\title{
One-Stage Repair and Reconstruction of Craniomaxillofacial Bone Defects
}

\author{
Jianhua Wang ${ }^{*}$, Chao Hu*, Gang Zhang, Songbo Qiu, Jun Cai, Xiaobo Wu, Zhao Xiang, \\ Yinghui Tan"
}

Department of Oral \& Maxillofacial Surgery, Xinqiao Hospital, Third Military Medical University, Chongqing, China. Email: "tanyh1962@yahoo.com.cn

Received October $11^{\text {th }}, 2012$; revised November $13^{\text {th }}$, 2012; accepted December $15^{\text {th }}, 2012$

\begin{abstract}
Objective: Severe craniomaxillofacial injuries and craniomaxillofacial tumors can lead to craniomaxillofacial bone defects and deformities. Seriously affect the patients' appearance and quality of life. So one-stage repair and reconstruction of craniomaxillofacial bone defects is of great significance. The current study summarizes the clinical experience of one-stage repair and reconstruction of craniomaxillofacial bone defects. Material and Methods: Data in one-stage repair and reconstruction of craniomaxillofacial bone defects performed on 13 patients were retrospectively analyzed out of 34 patients with craniomaxillofacial injuries or tumors who received treatment at the outpatient department between January 2002 and March 2011. Surgical indications and approaches were explored after two typical cases were detected. Results: One-stage repair and reconstruction of bone defects was suitable for patients with craniomaxillofacial injuries and excised craniomaxillofacial benign tumors. Adjacent autogenous bones and artificial materials (such as titanium plates, titanium mesh, and so on) work well for the repair of the craniomaxillofacial bone frame and restoration of facial features. Conclusions: Surgical indications should be strictly selected in one-stage repair and reconstruction of craniomaxillofacial bone defects and deformities. Furthermore, the adoption of autogenous bones and artificial materials is a good choice in restoring the craniofacial features.
\end{abstract}

Keywords: Craniomaxillofacial; Bone Defects; Repair and Reconstruction

\section{Introduction}

Severe craniomaxillofacial injuries and craniomaxillofacial tumors can lead to craniomaxillofacial bone defects and deformities. Considering bone defects and deformities caused by injuries or tumor resection have potential risks and the greatly influence the life quality of life of patients, the repair and reconstruction of defects during the early stages after the injury or soon after the surgery is of great significance [1,2]. However, craniomaxillofacial complex itself has a very complicated anatomic structure, which involves multiple anatomic regions, important structures, and organs (such as carotid arteries, veins, cranial nerves, and so on). Furthermore, during treatment, defects in the cerebral dura mater and soft tissues of the craniomaxillofacial complex, as well as the craniofacial frame and facial contour need to be repaired and reconstructed to prevent surgical complications. Meanwhile, the maxillofacial functions are protected or restored $[3,4]$. Thus, craniomaxillofacial surgery is a difficult and dangerous procedure with great clinical chal-

\footnotetext{
*The first two authors contributed equally to the work.

${ }^{\#}$ Corresponding author.
}

lenges, and requires interdisciplinary cooperation, including neurosurgery, oral and maxillofacial surgery, and so on [5,6]. Though immediate one-stage repair of craniomaxillofacial defects after injuries has great significance, the risks and the possible radiotherapy after resection of malignant craniomaxillofacial tumors forces surgeons to take into full consideration the indications for immediate postoperative one-stage repair [1,5], as well as the applicability of defect-repairing materials [7-9]. The current study summarizes 13 cases of one-stage repair and reconstruction of craniomaxillofacial bone defects.

\section{Data and Methods}

Between January 2002 and March 2011, 34 patients with craniomaxillofacial injuries or tumors received treatment at Xinqiao Hospital, Third Military Medical University, China, 23 of which were with bone injuries or benign tumors, of which 13 have accepted one-stage repair and reconstruction of craniomaxillofacial bone defects. Ten males and three females (aged 19 to 67 years old) were involved in the current study. Among them, 10 suffered from traffic accident injuries and 3 from ossifying fi- 
broma of craniofacial bones. All 10 patients having external injuries were diagnosed with frontal, temporal, jugal, zygomatic arch, and supramaxillary bone fractures. Considering the different degrees of combined craniocerebral injuries, treatments were given to the patients at the department of neurosurgery of local hospitals (no craniocerebral operation was done). After craniocerebral injury has stabilized, the patients were transferred into our department. Apart from routine examinations of the nervous system and the oromaxillofacial region, the patients were also subjected to craniofacial X-rays, computed tomography (CT), and three-dimensional (3D)-CT. ECT detection was carried out for patients with cerebrospinal leaks. Operations were performed under general anesthesia upon determination of the scope of craniofacial bone defects. All operations were performed using a trans-scalp coronal incision approach. For patients with external injuries, an extra incision through the vestibule of the oral cavity or at the infraorbital rim was made according to the site of maxillofacial fractures to expose the craniofacial injured region sufficiently. Reposition and fixation of the segments of maxillofacial fractures were performed, followed by craniotomy at the cranial fracture or diseased site. For patients with bone fractures, the fractured bones were repositioned and fixed, and for patients with bone defects, extra shape correction of the supraorbital rim was performed using titanic microplates. For one case of ossifying fibroma, the fragments from the cranial and supraorbital regions were corrected after tumor mass resection, repositioned after being boiled for $30 \mathrm{~min}$, and then fixed internally using titanium micro-plates. For the two cases of ossifying fibroma, the frontal and supraorbital defects were repaired with titanium mesh after tumor mass resection. Moreover, for cases with cerebrospinal rhinorrhea, the injured cerebral dura mater was repaired concurrently during the operation.

\section{Typical Case Report}

\subsection{Case 1}

A 36-year-old male patient was admitted to our hospital for treatment of right-sided craniomaxillofacial deformity, occlusal disturbance, and limitation of mouth opening after an external injury two months prior. The right side of the head and face were injured in a fall caused by a motorcycle accident, and stayed in a coma for more than 10 hours. The patient received treatment at a local hospital after emergency treatment, and the facial wounds were sutured after debridement. Upon admission to our hospital, the patient was conscious but with facial deformity (Figure 1(a)). Several soft tissue wound scars were present in his frontal, orbital, and zygomatic regions, and an orificium fistula formed in the supraorbital region with a drainage strip placed inside. The right orbital and zygomatic regions sunk and the eyeball shifted downward. The right eyeball was atrophied without light perception. CSF rhinorrhea was found and clear liquids exuded from the nasal cavity at intervals. His mouth opening was limited with the maximum opening of 1.0 $\mathrm{cm}$, and occlusal disturbance was found. Craniofacial Xray films, CT, and 3D-CT reconstruction displayed comminuted fractures, transposition of the fractured segments, and some bone loss at the right frontal, nasal, zygomatic, and supramaxillary bones (Figure 1(b)). ECT detection confirmed CSF rhinorrhea at the anterior cranial base (Figure 1(c)). The operation was performed after the absence of operative contraindications was confirmed through a whole-body examination. A combined approach of a trans-scalp coronal incision, an incision through the supramaxillary vestibule in the oral cavity, and an incision at the infraorbital rim was adopted to expose the fractured craniofacial segments (Figure 1(d)). The fractured zygomatic and supramaxillary segments were repositioned, and fixed internally using titanium microplates. Titanium micro-plates were molded according to the shape of the right supraorbital rim and fixed to reconstruct the contour of the orbit (Figure 1(e)). Craniotomy was performed at region parietalis of the right frontal bone. The bone flaps were turned open, the anterior skull base was isolated outside the dura mater, and the site of cerebrospinal rhinorrhea was repaired with fascia musculares. The frontal fragments and bone flaps were repositioned and fixed internally using titanium micro-plates (Figure 1(f)). After the operation, the cerebrospinal rhinorrhea disappeared, the maximum mouth opening and occlusion basically returned to normal, and the recovery of the patient's facial features was satisfactory (the patient refused to replace the atrophied eyeball with an artificial one) (Figures 1(g) and (h)).

\subsection{Case 2}

A 19-year-old male patient who suffered from left-sided facial deformity for more than ten years was hospitalized for treatment. On admission, the examinations showed that his left frontal and temporal regions bulged. The volume was $10 \mathrm{~cm} \times 8 \mathrm{~cm} \times 4 \mathrm{~cm}$ with an obscure boundary. His left eyeball protruded and shifted downward, but his vision and extraocular movements were normal (Figures 2(a) and (b)). X-ray films, CT, and 3DCT reconstruction showed expanded changes in the left frontal, temporal, and sphenoid bones, the frontal bone clearly protruded, and irregular cystic lesions were found in the mass (Figure 2(c)). Digital subtraction angiography showed that the left craniofacial blood vessels ran normally and no abnormal blood flow change was found. Based on the above results, he was diagnosed with left 


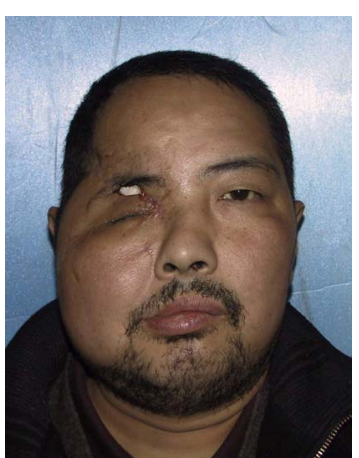

(a)

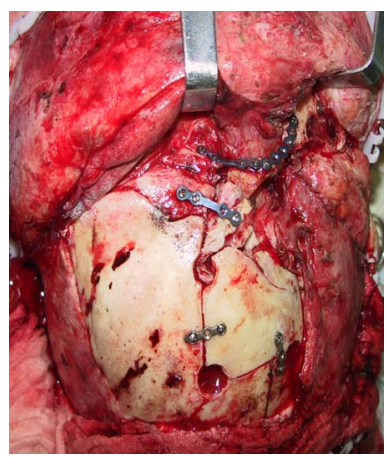

(e)

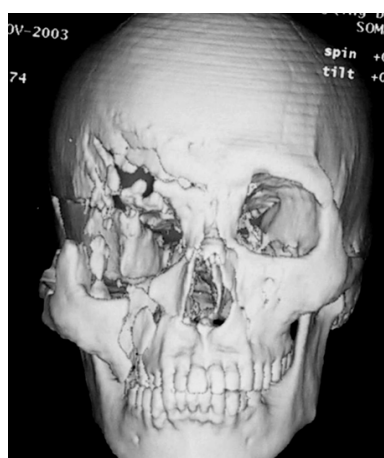

(b)

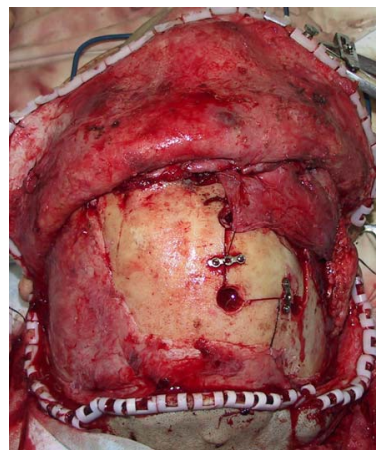

(f)

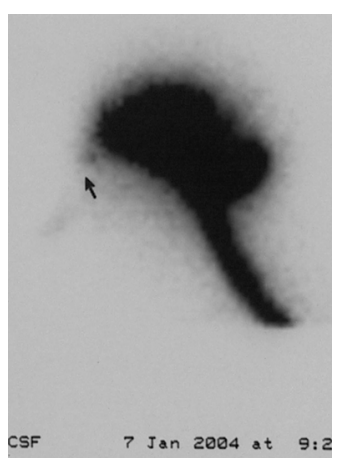

(c)

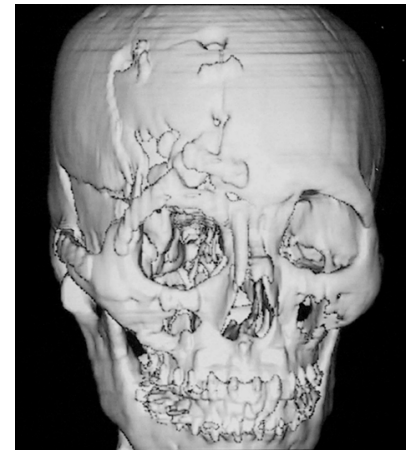

(g)

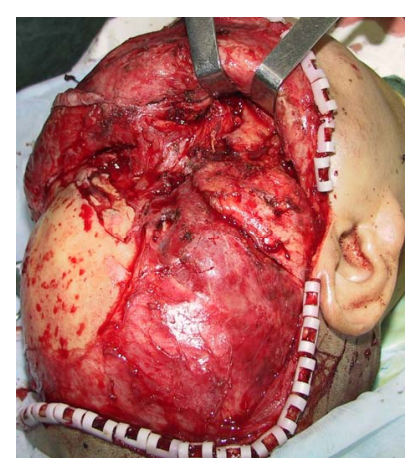

(d)

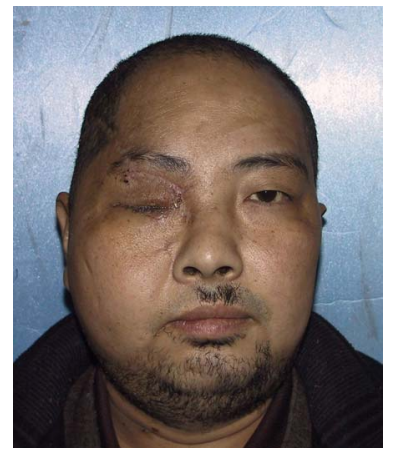

(h)

Figure 1. Case 1. The facial deformity before operation and detection by three-dimensional CT ((a) and (b)), and CSF rhinorrhea at the anterior cranial base detection by ECT (c). Operation process: expose the fracture and bone defect, repair the lesion area ((d)-(f)). The facial shape after operation and facial structure detection by three-dimensional CT ((g) and (h)).

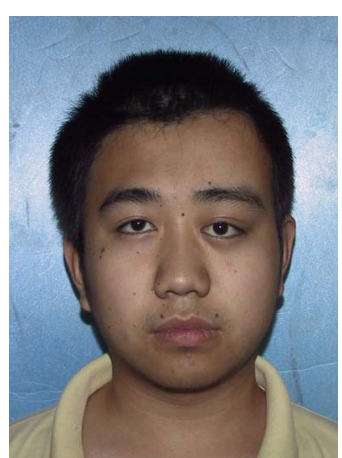

(a)

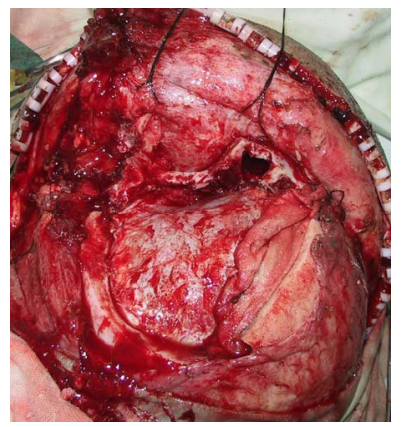

(e)

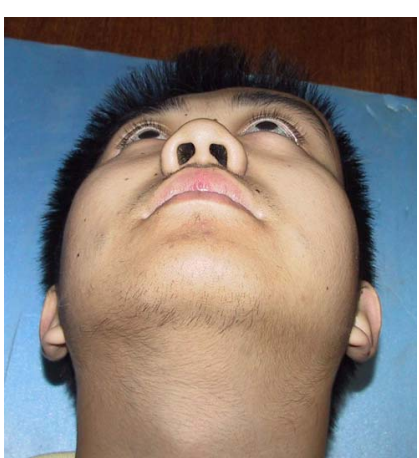

(b)

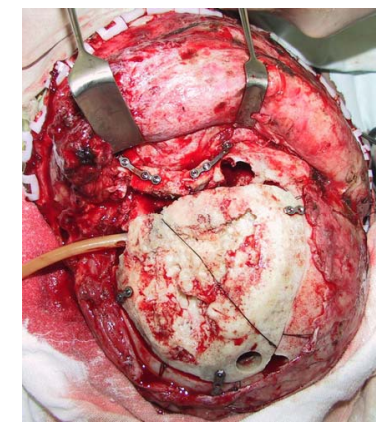

(f)

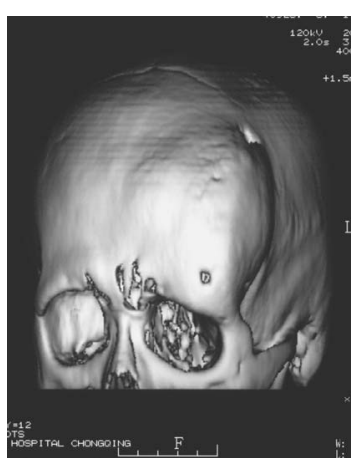

(c)

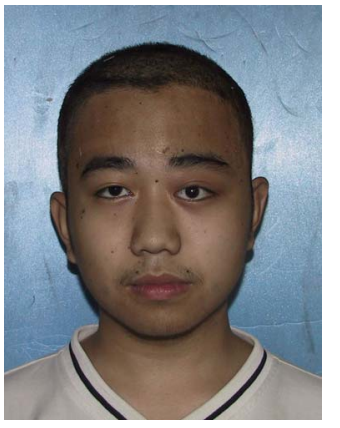

(g)

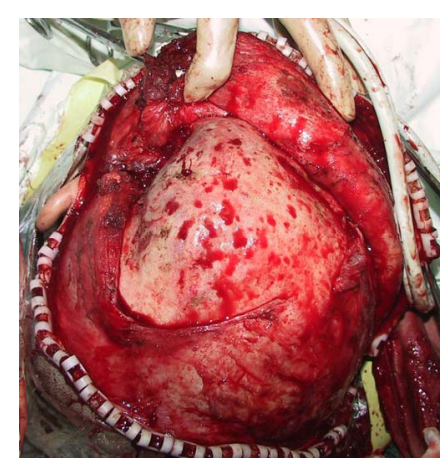

(d)

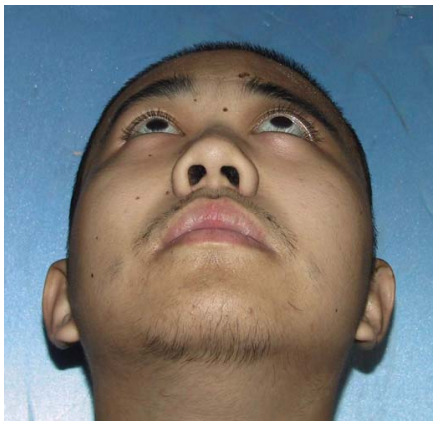

(h)

Figure 2. Case 2. The The craniofacial deformity before operation and detection by three-dimensional CT ((a)-(c)). Operation process: expose the tumor, tumor resection, repair the lesion area ((d)-(f)). The facial shape normal after operation ((g) and (h)). 
craniofacial ossifying fibroma. After confirmation of the absence of operative contraindications using whole-body examinations, the operation was performed under general anesthesia. A trans-scalp coronal incision approach was adopted to expose the frontal, temporal, and left supraorbital regions (Figure 2(d)). Osteotomy was performed around the mass after craniotomy. The fragments of the expanded frontal and supraorbital bones were taken out (Figure 2(e)), corrected according to morphologies of the contralateral frontal and supraorbital bones, boiled for $30 \mathrm{~min}$, repositioned, and fixed using titanium microplates (Figure 2(f)). After the operation, the patient had good wound healing and his facial features notably improved (Figures 2(g) and (h)). Examinations at two months after the operation showed satisfactory recovery of his facial features.

\section{Discussion}

The aims of craniomaxillofacial repair and reconstruction are to prevent the incidence of postoperative complications and to restore the craniofacial features and function $[1,2,10]$, in which the restoration of features and functions is the higher standard. Theoretically, all patients with deformities caused by craniomaxillofacial injury can undergo repair and reconstruction. Thus, craniomaxillofacial injuries can be considered as an operative indication for craniomaxillofacial bone repair and reconstruction. However, for patients with craniomaxillofacial tumors, one stage repair and reconstruction should be determined according to the nature of the tumor, its range, operative risks, and so on. The craniomaxillofacial complex has a very complicated anatomic structure wherein skull base tumors growing towards the maxillofacial complex or maxillofacial tumors invading the skull base can damage multiple anatomic regions and invade multiple important structures and organs. Complications are more likely with malignant tumors. Although theoretically, a malignant tumor can also be excised wholly around the normal tissues, achieving clinical radical excision when the tumor impinges or approaches important structures, such as the cavernous sinus, carotid arteries, optic nerves, and so on, is difficult. Under such conditions, performing one stage repair and reconstruction will be of no significance. On the contrary, such a procedure will likely increase operative risks and injuries. The better solution is to perform elective or postponed repair and reconstruction. However, whether an elective or postponed operation can be performed or not depends on the progression of the tumor based on the successive combined treatment such as radiotherapy, chemotherapy, and so on [11,12]. Although some studies have reported one-stage repair and reconstruction after malignant tumor resection, most have focused on either wound surface repair at the skull base with adjacent soft tissues or distant skin flaps, or repair of the cerebral dura mater to prevent cerebrospinal leakage $[3,4,13]$. Meanwhile, to prevent the incidence of postoperative complications in these operations, the connection between the cranial cavity and the nasal cavity is cut off. Thus, one stage repair is not considered suitable for bone defects after craniomaxillofacial malignant tumor resection. In the current study, five patients with craniomaxillofacial injuries and two with benign tumors were given concurrent one-stage repair and reconstruction of craniomaxillofacial bone defects during operation. Of the 14 patients with malignnant tumors, 8 received operative treatment, during which one stage repair was only restricted to repair of the defects of the cerebral dura mater and the skull base soft tissues.

In clinical practice, repair and reconstruction of craniomaxillofacial bone defects is one of the common problems for dentofacial surgeons, neurosurgeons, or plastic surgeons. A commonly used method involves determination of the range and contour of the craniomaxillofacial bone injuries and defects through CT detection and 3D-CT reconstruction [14]. A trans-scalp coronal incision approach with facial and oral vestibular incisions was adopted to expose the operative region extensively. Then, autogenous bone tissues (such as the cranial bone plates, the iliac bone, the ribs, and so on) or artificial materials (like titanic mesh, titanic plates, Medpor, etc.) are applied to repair the frame and configuration of the craniomaxillofacial bone defects, and to reconstruct the craniofacial features $[5,7,15,16]$. With the development of repair materials, repair methods and surgical techniques, theories, and methods for craniomaxillofacial repair and reconstruction have also rapidly developed [17]. Apart from basic experimental studies on the repair and regeneration of bone tissues through tissue engineering and stem cell techniques, clinical research on the application of individualized repair of craniomaxillofacial bones based on rapid prototyping techniques has also progressed, and some reports on its clinical application have been released. This method includes the following procedures: 3D imaging to reconstruct models of the craniomaxillofacial deformities or the pathologic changes, accurately determine the pathogenic condition and design an individualized operative treatment plan; based on computer-aided design and manufacture technique, individualized titanium prosthetic replacements for craniomaxillofacial bone defects are made; and then the replacements are surgically implanted into the bone defect region [18]. Although individualized craniofacial bone repair based on fast prototyping technique has the advantages of accurate repair and easy manipulation during operation, the cost is high, thus, it is not applied for most patients $[7,19,20]$. In addition, for a wide range of cra- 
niomaxillofacial bone defects or neoplastic lesions, especially lesions involving the cranial base or other complicated bone structures, mismatches between the prosthetic replacement designed in vitro and the actual ongoing condition are possible during the operation. When such mismatches occur, performing the operation according to the preoperative design is not advisable. The solution may be to perform proper repair and construction according to the intraoperative conditions combined with clinical experience based on preoperative detection, diagnosis, and design. For operations on wide-ranging craniomaxillofacial bone tumors, carrying out repair and reconstruction using autogenous and artificial materials based on the resection scope and the ongoing condition would be more practical [5,21]. Artificial bone is mainly applicable for patients with bone defects in large area, morphological recovery is better, but the cost is relatively expensive. Autogenous bone is mainly applicable for patients with craniofacial benign tumors, and it is easily obtained, but is not suitable for patients with bone defects in large area. In the current study, the craniofacial fragments from the patients with craniofacial injuries were set aside. These fragments were repositioned and fixed, and the defects were repaired using adjacent autogenous bone or artificial materials. For patients with craniofacial tumors, to maintain intraoperative security, the tumor was excised with ample surgical margins and the craniofacial frame was reconstructed through shape correction of the wide-ranging cranial bone defects after physical inactivation of the pathologic fragments, as well as repair using artificial materials. Good outcomes were achieved in all patients. Wounds in all patients healed after the one-stage operation without complications, and the recovery of their craniofacial features were satisfactory.

In summary, the operative indications should be strictly selected for the determination of one-stage repair and reconstruction of craniofacial bone defects and deformities before the operation. One-stage repair and reconstruction is mainly applicable for patients with craniofacial injuries and benign tumors. Furthermore, the use of autogenous bones from the injured region or the adjacent regions assisted with artificial materials is safe, economical, and practical to for repair and reconstructtion.

\section{REFERENCES}

[1] J. T. Goodrich, "Craniofacial Surgery: Complications and Their Prevention,” Seminars in Pediatric Neurology, Vol. 11, No. 4, 2008, pp. 288-300. doi:10.1016/j.spen.2004.10.001

[2] E. Neovius and T. Engstrand, "Craniofacial Reconstruction with Bone and Biomaterials: Review over the Last 11 Years," Journal of Plastic, Reconstructive \& Aesthetic
Surgery, Vol. 63, No. 10, 2010, pp. 1615-1623. doi:10.1016/j.bjps.2009.06.003

[3] D. Bondin and G. L. Ross, "The Use of a Combined Radial Forearm Flap and Radial Fascial Flap for Layered Dural Lining and an Orbital Defect Reconstruction,” Journal of Plastic, Reconstructive \& Aesthetic Surgery, Vol. 64, No. 7, 2011, pp. e167-e169.

[4] F. P. Fechner and D. G. Deschler, "Microvascular Free Flap Reconstruction after Craniofacial Trauma," Operative Techniques in Otolaryngology-Head and Neck Surgery, Vol. 13, No. 4, 2002, pp. 309-315.

[5] J. J. Kuttenberger and N. Hardt, "Long-Term Results Following Reconstruction of Craniofacial Defects with Titanium Micro-Mesh Systems," Journal of Cranio-Maxillofacial Surgery, Vol. 29, No. 2, 2001, pp. 75-81. doi:10.1054/jcms.2001.0197

[6] L. Pereira, M. A. Carron and R. H. Mathog, "Traditional Craniofacial Resection,” Operative Techniques in Otolaryngology—Head and Neck Surgery, Vol. 21, No. 1, 2010, pp. 2-8. doi:10.1016/j.otot.2009.06.004

[7] U. Klammert, U. Gbureck, E. Vorndran, et al., "3D Powder Printed Calcium Phosphate Implants for Reconstruction of Cranial and Maxillofacial Defects," Journal of Cranio-Maxillofacial Surgery, Vol. 38, No. 8, 2010, pp. 565-570. doi:10.1016/j.jcms.2010.01.009

[8] F. J. Kramer, B. Sinikoviv, M. Mueller, et al., "Experimental Application of a Bifocal Transport Osteogenesis for Craniofacial Reconstruction,” Journal of Cranio-Maxillofacial Surgery, Vol. 36, No. 4, 2008, pp. 218-226. doi:10.1016/j.jcms.2007.12.001

[9] D. Marchac and A. Greensmith, "Long-Term Experience with Methylmethacrylate Cranioplasty in Craniofacial Surgery," Journal of Plastic, Reconstructive \& Aesthetic Surgery, Vol. 61, No. 7, 2008, pp. 744-752.

[10] G. Cantù, C. L. Solero, N. Pizzi, et al., "Skull Base Reconstruction after Anterior Craniofacial Resection,” Journal of Cranio-Maxillofacial Surgery, Vol. 27, No. 4, 1999, pp. 228-234. doi:10.1016/S1010-5182(99)80034-1

[11] C. S. Chang, L. Bergeron, C. C. Liao, et al., "Craniofacial Reconstruction of Primary Osteogenic Sacoma of the Skull," Journal of Plastic, Reconstructive \& Aesthetic Surgery, Vol. 63, No. 8, 2010, pp. 1265-1268.

[12] M. Lotfy, R. Xu, M. Mcgirt, et al., "Reconstruction of Skull Base Defects in Sphenoid Wing Dysplasia Associated with Neurofibromatosis I with Titanium Mesh," Clinical Neurology and Neurosurgery, Vol. 112, No. 10, 2010, pp. 909-914.

[13] B. G. Bentz, M. H. Bilsky, J. P. Shah and D. Kraus, “Anterior Skull Base Surgery for Malignant Tumors: A Multivariate Analysis of 27 Years of Experience,” Head Neck, Vol. 25, No. 7, 2003, pp. 515-520. doi:10.1002/hed.10250

[14] C. H. Buitrago-Téllez, W. Schilli, M. Bohnert, et al., “A Comprehensive Classification of Craniofacial Fractures: Postmortem and Clinical Studies with Two- and ThreeDimensional Computed Tomography," Injury, Vol. 33, No. 8, 2002, pp. 651-668. doi:10.1016/S0020-1383(02)00119-5

[15] J. J. Mendonca-Caridad, P. Juiz-Lopez and J. P. Rubio- 
Rodriguez, "Frontal Sinus Obliteration and Craniofacial Reconstruction with Platelet Rich Plasma in a Patient with Fibrous Dysplasia,” International Journal of Oral and Maxillofacial Surgery, Vol. 33, No. 1, 2006, pp. 8891. doi:10.1016/j.ijom.2005.06.017

[16] D. N. Silva, M. G. Oliveira, E. Meurer, et al., "Dimensional Error in Selective Laser Sintering and 3D-Printing of Models for Craniomaxillary Anatomy Reconstruction,” Journal of Cranio-Maxillofacial Surgery, Vol. 36, No. 8, 2008, pp. 443-449. doi:10.1016/j.jcms.2008.04.003

[17] K. A. Matthew and M. J. Lawrence, "New Developments in Craniofacial Reconstruction," Current Opinion in Otolaryngology \& Head and Neck Surgery, Vol. 10, No. 4, 2002, pp. 282-285.

doi:10.1097/00020840-200208000-00007

[18] A. Müller, K. G. Krishnan, E. Uhl, et al., "The Application of Rapid Prototyping Techniques in Cranial Reconstruction and Preoperative Planning in Neurosurgery,”
Journal of Craniofacial Surgery, Vol. 14, No. 6, 2003, pp. 899-914. doi:10.1097/00001665-200311000-00014

[19] W. Tang, J. Long, F. Feng, et al., "Condyle Replacement after Tumor Resection: Comparison of Individual Prefabricated Titanium Implants and Costochondral Grafts," Oral Surgery, Oral Medicine, Oral Pathology, Oral Radiology and Endodontology, Vol. 108, No. 2, 2009, pp. 147-152. doi:10.1016/j.tripleo.2009.01.028

[20] W. Tang, L. Guo, J. Long, et al., "Individual Design and Rapid Prototyping in Reconstruction of Orbital Wall Defects," Journal of Oral and Maxillofacial Surgery, Vol. 68, No. 3, 2010, pp. 562-570. doi:10.1016/j.joms.2009.04.042

[21] K. E. Salyer, C. R. Barcelo and Y. C. Por, "Extensive Neglected Psammomatoid Ossifying Fibroma with Craniofacial Deformity," Journal of Craniofacial Surgery, Vol. 15, No. 6, 2004, pp. 1033-1039. doi:10.1097/00001665-200411000-00031 